\title{
Profile of a Galactic Spherical Cloud of Self-Gravitating Fermions
}

\author{
B. G. Giraud* and R. Peschansk \\ Institut de Physique Théorique, Centre d'Etudes Saclay, 91191 Gif-sur-Yvette, France
}

\begin{abstract}
The field which binds a thermal fermionic cloud is defined as a Hartree integral upon its density. In turn, the density results from the field via a Thomas-Fermi occupation of the local phase space. This defines a complete theory of all properties and observables for the cloud. As an application to dark matter halos, comparisons with astronomic data on dwarf spheroidal galaxies are provided and discussed. Estimates of the elementary fermion mass are obtained, serving as a phase-space bound on fermionic dark matter.
\end{abstract}

PACS numbers: 95.35.+d 95.30.Cq 98.52.Wz

\section{INTRODUCTION}

While there is strong evidence for the existence of dark matter, the absence of its direct detection triggers many questions, such as i) are we talking about light or heavy particles? ii) are they fermions, such as, for instance, sterile neutrinos, or a new kind of BSM fermions, or are they some bosons? iii) are they self-binding into balls by gravitation only or is there a contribution from some additional interaction between the dark particles? iv) what could be the mass of a dark particle? and so on.

The present work deals with the questions of selfbinding and elementary mass, under very simple hypotheses, namely a fermionic nature of the particles, in non relativistic thermal equilibrium, and a self-consistent gravitational field. Recently, such a hypothesis has been explored for dark matter made of sub- $\mathrm{keV}$ degenerate fermions [1, 2] (see also [3] which considers a degenerate core surrounded by a thermal envelope). In the following we consider the case of quasi-degenerate fermions, meaning that the temperature to be considered is low enough such that the quantum effects have to be taken into account together with the temperature. For the same reason we are led to consider fermions presently in a nonrelativistic thermal state. The comparison with data is made for fermionic clouds of dark matter supposed to be associated with the known Dwarf Spheroidal Galaxies (DSG) in the vicinity of the Milky Way [4, 5].

More generally, and independently from this precise hypothesis, the determination of the properties of thermal fermionic galactic clouds proved to be useful to determine model-independent phase-space lower bounds on the mass of fermionic dark matter, starting with the pioneering Ref. [6] which considered an initial thermal relativistic stage of the fermion cloud. The bound is due to the existence of a maximal phase-space density which stays valid after further collision-less and dissipation-less evolution, leading to a quite model-independent lower mass bound for the fermion. Various phase-space bounds related to this method have been discussed and evaluated by different ways since then, noticeably in Refs. 7-10].

In our scheme, since our calculations define a complete theory of all properties and observables for the cloud, depending on its temperature, the determination (or the lower bound) of an allowed elementary fermion mass from the cloud finds a natural application to the problem of the elementary fermion mass using DSG data.

We will here define the gravitational field by a simple Hartree convolution integral between the basic Newton attraction and the dark matter density. In turn, this density will result from the field according to a ThomasFermi approximation. This allows a closing of the algebra into a self-consistent equation for the field, or a self-consistent equation for the density. Such equations are reminiscent of the Poisson equation and can be easily solved numerically. We thus obtain a complete description of the profile of a dark object, and of properties estimated by astronomic observations, such as velocity dispersion and radial extension. Our description is parametrized by five parameters, namely Planck's constant $h$, the Newton gravitational constant $G$, the elementary dark particle mass $m$, a temperature $T$ and a scale parameter, that turns out to be a non-relativistic velocity $v_{0}$. Then the simple structure of our theory provides a formula that calculates the elementary mass $m$ in terms of the other parameters and of astronomic observations.

The equations obeyed by a self-gravitating fermionic cloud at a temperature $T$ are displayed in Section II. They provide a rich zoology of density profiles and related observables, via both analytical and numerical approaches. Section III is a numerical application of our results to astronomic cases. It shows somewhat realistic numbers, on the galactic scale. We will even find a glimpse of an answer to the question of the elementary masses, leading to an evaluation of a lower mass bound on a dark fermion, when combining the data on the various DSG's. Finally Sections IV and V offer a discussion and conclusion. 


\section{BASIC EQUATIONS AND FORMAL CONSEQUENCES}

Spherical symmetry is assumed throughout this paper. In a first part, corresponding to degenerate fermions $(\mathrm{T}=0)$, we also assume (and indeed check) that the dark matter cloud has a finite radius $R$. The dark particles, assumed to be spin $\frac{1}{2}$ fermions with mass $m$, are bound by their self-consistent potential field $\phi$. Let $\vec{r}$ mean the position coordinates and set $r \equiv|\vec{r}|$. Then,

$$
\phi(r)=-G \int d^{3} \overrightarrow{r^{\prime}} \frac{\rho\left(r^{\prime}\right)}{\left|\vec{r}-\overrightarrow{r^{\prime}}\right|},
$$

also reads,

$$
\frac{1}{4 \pi G} \phi(r)=-\frac{1}{r} \int_{0}^{r} d r^{\prime} r^{\prime 2} \rho\left(r^{\prime}\right)-\int_{r}^{R} d r^{\prime} r^{\prime} \rho\left(r^{\prime}\right) .
$$

Here, $G$ is the gravitational constant and $\rho\left(r^{\prime}\right)$ is the mass density of the dark matter inside the spherical shell contained between radii $r^{\prime}$ and $r^{\prime}+d r^{\prime}$. The first term in the right hand side of Eq.(2) accounts for the mass internal to the sphere with radius $r$. The second term accounts for the mass in the "corona", between $r$ and $R$.

At the surface, the field reaches the value, $\phi(R)=$ $-G M / R$, where $M$ is the total mass, $M \equiv M(R)=$ $4 \pi \int_{0}^{R} d r^{\prime} r^{\prime 2} \rho\left(r^{\prime}\right)$. Above the surface, $\phi$ continues as, $\phi(r)=-G M / r$, but, in the following, we shall be concerned with internal properties only, namely $0 \leq r \leq R$.

The centripetal force reads, upon derivating Eq.(2),

$$
-\frac{d \phi}{d r}=-\frac{G M(r)}{r^{2}}, \quad M(r)=4 \pi \int_{0}^{r} d r^{\prime} r^{\prime 2} \rho\left(r^{\prime}\right) .
$$

The field $\phi$, an increasing function of $r$, is negative $\forall r$.

It can be noticed, incidentally, that the definition of $\phi$ from Eq.(11) makes $\phi$ be a Hartree potential [1]. In principle, one should also consider a Fock potential [12], the result of exchange terms in the fermionic gravitational interaction. This Fock term is neglected here.

With the well known, Thomas-Fermi approximation [13], the fermionic population of each volume element of this large system is assumed, at zero temperature, to make a Fermi sphere in momentum space, with some radius $p_{F}(r)$. Accordingly, the mass density reads,

$$
\rho(r)=m \frac{8 \pi}{3 h^{3}}\left[p_{F}(r)\right]^{3},
$$

where $h$ is Planck's constant and $m$ is the dark matter particle mass. At position $r$ the Fermi momentum, $p_{F}(r)$, is defined from a maximal energy level, $m \mu$, available for occupation,

$$
\frac{p_{F}^{2}(r)}{2 m}+m \phi(r)=m \mu,
$$

where the product, $m \mu$, defines a chemical potential. That radius $R$, where $\phi$ equates $\mu$, induces $p_{F}(R)=$
0, hence the "local Fermi sphere" shrinks to zero and the Thomas-Fermi approximation experiences difficulties with a turning point. In practice for the present theory, the density, $\rho(R)$, vanishes according to Eq.(4) since then $p_{F}=0$. This defines the surface as we see it. Accordingly, we set $\mu=-G M / R$.

Let us now consider a cloud in a thermal equilibrium, the likely result of a cosmological period when dark matter was not yet decoupled. For a finite temperature $T$ the Fermi sphere at position $r$ is partly filled only, and there are "compensating" fermions above the Fermi surface, both facts described by the well known Fermi occupation formula,

$$
\rho=\frac{8 \pi m}{h^{3}} \int_{0}^{\infty} p^{2} d p \frac{1}{1+\exp \left\{\left[\frac{p^{2}}{2 m}+m \psi\right] /(k T)\right\}},
$$

with $k$ the Boltzmann constant and $\psi \equiv \phi-\mu$. We might still define the surface by the condition,

$$
\psi(R)=0,
$$

but, above that surface, namely when $\psi$ becomes positive, the formula, Eq.(6), allows for residual fermions. Their probability in momentum space will become exponentially small, but a density tail occurs in coordinate space. It is easy to verify that, actually, the obtained solutions tell that $\psi(r)$ is of order $\log r$ and $\rho(r)$ of order $r^{-2}$, when $r \rightarrow \infty$. Hence a divergence occurs for the total mass, another concern with the validity of a ThomasFermi approach if one uses it too brutally. For the sake of caution and rigor, we shall present in the following, when necessary, two sets of results at least, namely i) a set where integrals upon $r$ obtain from a cut-off at $R$ as defined by Eq.(7), then ii) a set where integrals are extended until $1.5 R$, for example, or more, to show tail effects. (One might also argue that a momentum cut-off is necessary to avoid relativistic situations, but we will stay in the non-relativistic regime.)

It is now convenient to introduce a velocity $v_{0}$ such that $\psi_{0} \equiv \psi(0)=-v_{0}^{2} / 2$, then the scalings, $p=m v_{0} q$ and $\psi(r)=v_{0}^{2} \chi(r) / 2$, where $q$ and $\chi$ become dimensionless. Then $\chi_{0} \equiv \chi(0)=-1, \chi(R)=0$ and Eq.(6) reads,

$$
\rho(r)=\frac{8 \pi m^{4} v_{0}^{3}}{h^{3}} \int_{0}^{\infty} \frac{q^{2} d q}{1+\exp \left\{\left[q^{2}+\chi(r)\right] / \eta\right\}},
$$

where $\eta=k T /\left(m v_{0}^{2} / 2\right)$. In the following, we shall only consider cases where $\eta<1$, for the obvious reason that quantum effects might be washed out if the thermal energy order of magnitude, $k T$, exceeds the kinetic energy order of magnitude, $m v_{0}^{2} / 2$.

The density also reads,

$$
\rho(r)=-\frac{2(\pi \eta)^{\frac{3}{2}} m^{4} v_{0}^{3}}{h^{3}} L i\left[\frac{3}{2},-e^{-\chi(r) / \eta}\right],
$$


where $L i$ is the polylogarithm function. Define now,

$$
\mathcal{L}_{3}(\chi, \eta) \equiv 3 / 4 \sqrt{\pi} \eta^{\frac{3}{2}} L i\left[\frac{3}{2},-e^{-\chi / \eta}\right] .
$$

Notice that,

$$
\lim _{\eta \rightarrow 0} \mathcal{L}_{3}(\chi, \eta)=-(-\chi)^{\frac{3}{2}},
$$

making easier the transition with the degenerate fermion case. Moreover we find

$$
\rho(r)=-\frac{8 \pi m^{4} v_{0}^{3}}{3 h^{3}} \mathcal{L}_{3}(\chi, \eta) .
$$

The second of Eqs.(3) then becomes,

$$
M(r)=-\frac{32 \pi^{2} m^{4} v_{0}^{3}}{3 h^{3}} \int_{0}^{r} d r^{\prime} r^{\prime 2} \mathcal{L}_{3}\left[\chi\left(r^{\prime}\right), \eta\right] .
$$

Since $d \phi / d r$ and $d \psi / d r$ are trivially equal, because $\mu$ is a constant, the first of Eqs.(3) reads,

$$
-\frac{d \chi}{d r}=\frac{64 \pi^{2} m^{4} v_{0} G}{3 h^{3} r^{2}} \int_{0}^{r} d r^{\prime} r^{\prime 2} \mathcal{L}_{3}\left[\chi\left(r^{\prime}\right), \eta\right],
$$

where we again used the scaling relating $\psi$ and $\chi$. A further derivative yields,

$$
\frac{1}{r^{2}} \frac{d}{d r} r^{2} \frac{d \chi}{d r}=-K_{0} \mathcal{L}_{3}[\chi(r), \eta],
$$

with

$$
K_{0}=\frac{64 \pi^{2} m^{4} v_{0} G}{3 h^{3}} .
$$

This Eq.(15) is actually the Poisson equation for the present problem. Define now a length scale $r_{0}$ by the condition, $r_{0}^{2} K_{0}=1$, namely

$$
r_{0}=\frac{\sqrt{3} h^{\frac{3}{2}}}{8 \pi m^{2} \sqrt{v_{0} G}} .
$$

Then implement the scalings, $r=r_{0} x, R=r_{0} X$, and $\chi(r)=\xi\left(r / r_{0}\right)$. This yields,

$$
\frac{1}{x^{2}} \frac{d}{d x} x^{2} \frac{d \xi}{d x}=-\mathcal{L}_{3}[\xi(x), \eta] .
$$

This simplifies into, $\frac{1}{x^{2}} \frac{d}{d x} x^{2} \frac{d \xi}{d x}=[-\xi(x)]^{\frac{3}{2}}$, for $\eta=0$.

We show in Figure (11) the solutions $\xi(x)$ numerically obtained for $\eta=0, .1, .3 ., .5$, respectively. We used the following boundary conditions, $\xi(0)=-1$ and $d \xi /\left.d x\right|_{x=0}=0$. The latter means that the field has a smooth minimum at the center of the dark ball.

Then we show, with a sequence of dots in Figure (2), the evolution in terms of $\eta$ of the "scaled radius" $X$ where $\xi$ vanishes. This is of interest if the physical scale of the ball radius is defined as, $R=r_{0} X$. It is seen that $X$ diminishes when $\eta$ increases. The same Fig.(2)

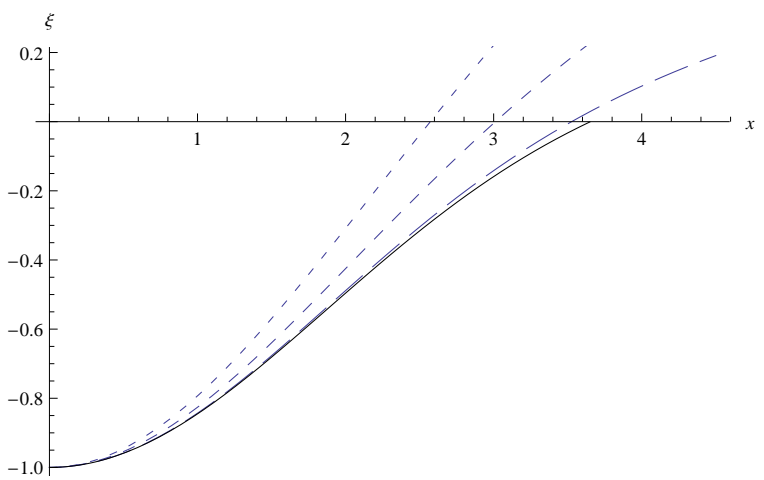

FIG. 1. Scaled profiles $\xi$. Full line for $\eta=0$, long dashes for $\eta=.1$, moderate dashes for $\eta=.3$, short dashes for $\eta=.5$.

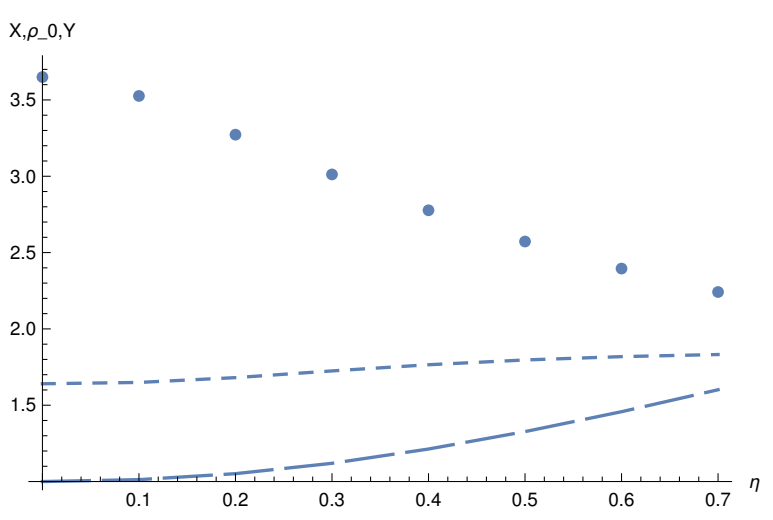

FIG. 2. Evolutions in terms of $\eta$. Dots for the root $X$ of profile $\xi$, long dashes for the density $\rho(0)$ at center and short dashes for the radius $Y$ at mid-density.

shows (long dashes) that the density at the origin increases, and (short dashes) that the radius, $Y$, where the density is half the density at center, $\rho(Y)=\rho(0) / 2$, increases, but slightly only. Notice that such conclusions, and most forthcoming ones, are valid only for a fixed value of $v_{0}$ when $\eta$ changes. Cases with different values of $v_{0}$ cannot be so trivially compared, since, given $m$, the present model of a dark ball is driven by two independent physical parameters, namely $v_{0}$ and the temperature, $T=\left(m v_{0}^{2} / 2\right) \eta / k$. Then $T$ occurs in $\eta$ only but $v_{0}$ influences both $\eta$ and $r_{0}$. In Figure (3), we compare densities. The comparison is done for normalized profiles, $\rho_{n}(x) \equiv \rho(x) / \rho(0)$. The full line corresponds to $\eta=0$ and the long, moderate and short dashes to $\eta=.1, .3, .5$, respectively. Tails grow when $\eta$ increases.

As is seen in Figure (44), the trend of the total mass is not the same whether one calculates it with integrals limited to $X$ or extended to $1.5 X$. In the first case, $M$ first increases, then decreases as a function of $\eta$. The second case cannot return a mass including tail effects for $\eta=0$, since there is no tail. A systematic increase is then found for finite values of $\eta$.

Of special interest for our subject is the traditional 


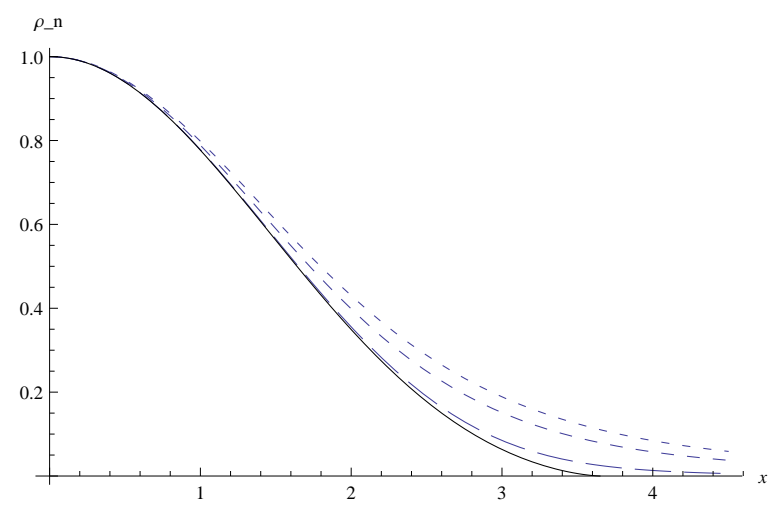

FIG. 3. Renormalized densities $\rho_{n}(x)$. Full line, $\eta=0$. Long, moderate, short dashes for $\eta=.1, .3, .5$. respectively.

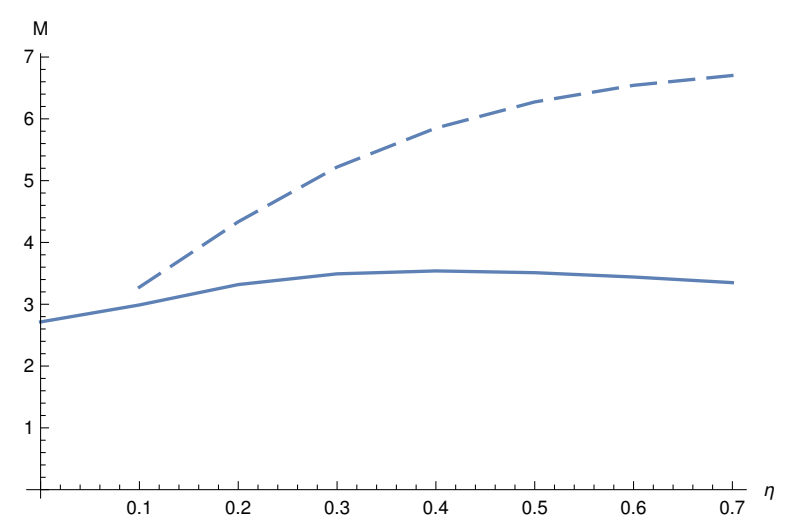

FIG. 4. Total mass as a function of $\eta$. Full line for the mass interior to the node of $\psi$, dashes for the mass including part of the tail.

observation [14] of the radial velocity dispersion for stars and the cosmological hypothesis that the star dispersion is equal to that of the dark matter particles. Since in our Thomas-Fermi scheme the phase space density reads,

$$
\rho_{p s}(\vec{r}, \vec{p}) d \vec{r} d \vec{p}=\frac{2 h^{-3} d \vec{r} d \vec{p}}{1+\exp \left\{\left[\frac{p^{2}}{2 m}+m \psi\right] /(k T)\right\}},
$$

the radial velocity dispersion for the dark particles is, inside a ball of radius $R$,

$$
\left\langle\frac{p^{2}}{3 m^{2}}\right\rangle=\frac{32 \pi^{2}}{3 h^{3} m^{2}} \int_{0}^{R} \int_{0}^{\infty} \frac{r^{2} d r p^{4} d p}{1+\exp \left\{\left[\frac{p^{2}}{2 m}+m \psi\right] /(k T)\right\}},
$$

namely, after now familiar scalings, $p=q m v_{0}, r=x r_{0}$, $R=X r_{0}$ and $\psi=\xi\left(r / r_{0}\right) v_{0}^{2} / 2$,

$$
\left\langle\frac{p^{2}}{3 m^{2}}\right\rangle=-\mathcal{K} v_{0}^{2} \int_{0}^{X} x^{2} d x \text { Li }\left\{\frac{5}{2},-\exp \left[\frac{-\xi(x)}{\eta}\right]\right\} \text {. }
$$

with $\mathcal{K}=4(\pi \eta)^{\frac{5}{2}}\left(r_{0} m v_{0} / h\right)^{3}$. Actually, it may be more interesting to use here the function,

$$
\mathcal{L}_{5}(\xi, \eta) \equiv \frac{15 \sqrt{\pi} \eta^{\frac{5}{2}}}{8} L i\left[\frac{5}{2},-e^{-\frac{\xi}{\eta}}\right]
$$

with the advantage that $\lim _{\eta \rightarrow 0} \mathcal{L}_{5}(\xi, \eta)=-(-\xi)^{\frac{5}{2}}$. Then Eq.(21) becomes

$$
\left\langle\frac{p^{2}}{3 m^{2}}\right\rangle=-\frac{32 \pi^{2}\left(m v_{0} r_{0}\right)^{3} v_{0}^{2}}{15 h^{3}} \int_{0}^{X} x^{2} d x \mathcal{L}_{5}[\xi(x), \eta] .
$$

Note that the particle number reads,

$$
N=\frac{32 \pi^{2}}{h^{3}} \int_{0}^{R} \int_{0}^{\infty} \frac{r^{2} d r p^{2} d p}{1+\exp \left\{\left[\frac{p^{2}}{2 m}+m \psi\right] /(k T)\right\}},
$$

or, as well,

$$
N=-\frac{32 \pi^{2}\left(m v_{0} r_{0}\right)^{3}}{3 h^{3}} \int_{0}^{X} x^{2} d x \mathcal{L}_{3}[\xi(x), \eta] .
$$

At $T=0$, Eq.(20) reduces to,

$$
\left\langle\frac{p^{2}}{3 m^{2}}\right\rangle=\frac{128 \sqrt{2} \pi^{2} m^{3}}{15 h^{3}} \int_{0}^{R} r^{2} d r \psi(r)^{\frac{5}{2}},
$$

hence, after scaling,

$$
\left\langle\frac{p^{2}}{3 m^{2}}\right\rangle=\frac{32 \pi^{2}\left(m v_{0} r_{0}\right)^{3} v_{0}^{2}}{15 h^{3}} \int_{0}^{X} x^{2} d x \xi(x)^{\frac{5}{2}}, X \simeq 3.6537 .
$$

Similarly, at $T=0$,

$$
N=\frac{32 \pi^{2}\left(m v_{0} r_{0}\right)^{3}}{3 h^{3}} \int_{0}^{X} x^{2} d x \xi(x)^{\frac{3}{2}}, X \simeq 3.6537 .
$$

For each value of $r$, or of the scaled radius $x$, we may define, from the previous formulas, a local average radial dispersion, $\mathcal{L}_{5} / \mathcal{L}_{3}$, where we discarded all inessential coefficients. We show in Figure (5), from top to bottom, the corresponding curves for $\eta=.7, .5, .3, .1$, respectively. The length of dashes increases as $\eta$ diminishes. The full line in Figure (5) shows the limit ratio, $-\xi$, corresponding to $T=0$. The line stops beyond the sharp surface radius, $X=3.6537$, naturally. Note how all finite temperature curves seem to stabilize when $x$ exceeds that critical value, 3.6537. This might help defining empirically a "surface" for the ball, as a transition between a "core" and a "tail", typically at a radius of order, $R \sim 4 r_{0}$.

From Eq.(23) and Eq.(25), the radial dispersion, when averaged over the whole ball, reads

$$
\Delta \equiv \sigma^{2} \equiv\left\langle\frac{p^{2}}{3 m^{2}}\right\rangle / N=\frac{v_{0}^{2}}{5} \frac{\int_{0}^{X} x^{2} d x \mathcal{L}_{5}(\xi, \eta)}{\int_{0}^{X} x^{2} d x \mathcal{L}_{3}(\xi, \eta)},
$$

where, for each $\eta$, we take $X$ as the root of $\xi$ or extend $X$ to 1.5 that root. For the case, $T=0, \mathcal{L}_{5}$ and $\mathcal{L}_{3}$ are replaced by their now familiar limits and, obviously, there is no "extended integral" result. In Figure (6), where the coefficient, $v_{0}^{2} / 5$, is voluntarily omitted, the results of this averaging of the radial velocity dispersion over the ball volume are shown by a full line for integrals limited by the field roots and by dots for "extended integrals". It will 


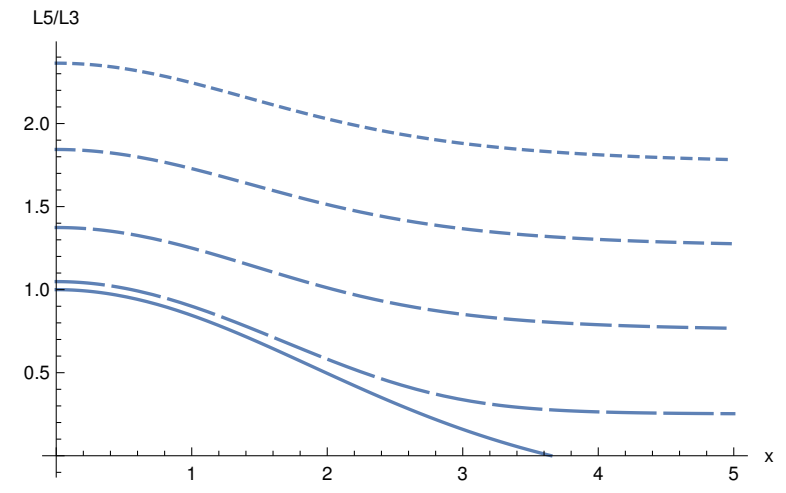

FIG. 5. Profiles of mean square radial velocities. Full line, $\eta=0$. Dashes shorten as $\eta=.1, .3, .5, .7$.

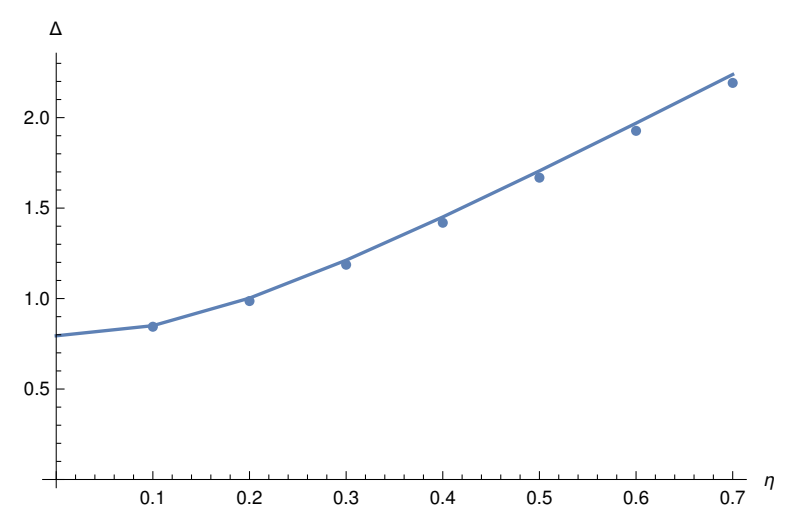

FIG. 6. Mean square radial velocity in terms of $\eta$. Full line for integrals interior to the node of $\phi-\mu$, dots for including part of the tail.

be noted that tail effects seem to be small for the present observable. But, as might be expected, the dispersion increases as a function of $\eta$.

Another concept used in galactic astronomy [14] is the surface density, $\hat{\rho}(s)$, defined as follows. Consider an equatorial plane of the dark ball and a (positive or negative) height $z$ measured from that plane. Coordinates inside the plane may be chosen as polar coordinates, $\{s, \theta\}$ and the triplet, $\{s, \theta, z\}$, make a simple set of cylindrical coordinates. Actually, the angle, $\theta$, will be irrelevant in the following. The radius from the ball center reads, obviously, $r=\sqrt{s^{2}+z^{2}}$. Given a radius $R$ for the ball, the surface density is then defined as,

$$
\hat{\rho}(s)=2 \int_{0}^{\sqrt{R^{2}-s^{2}}} d z \rho\left(\sqrt{s^{2}+z^{2}}\right) .
$$

Upon taking advantage of Eq.(9), this becomes, after an obvious scaling of the form, $R=r_{0} X, z=r_{0} \zeta$ and $s=$ $r_{0} \sigma$

$$
\hat{\rho}\left(r_{0} \sigma\right)=-\frac{8 \pi m^{4} v_{0}^{3} r_{0}}{3 h^{3}} \int_{0}^{Z(\sigma)} d \zeta \mathcal{L}_{3}\left[\xi\left(\sqrt{\sigma^{2}+\zeta^{2}}\right), \eta\right]
$$

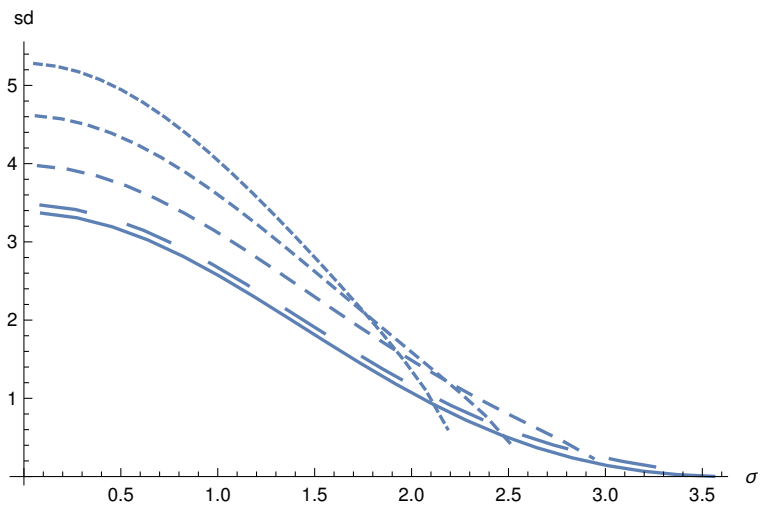

FIG. 7. Surface densities when the radii are defined by the roots of $\xi$. Full line, $\eta=0$. Dashes shorten as $\eta=.1, .3, .5, .7$.

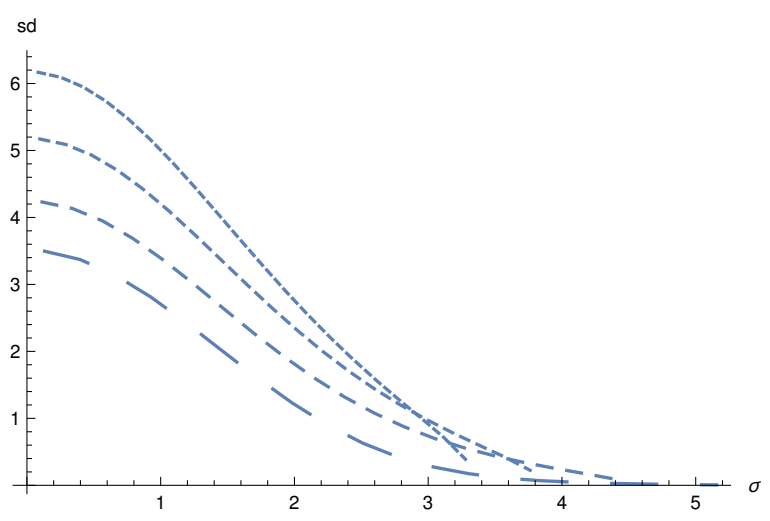

FIG. 8. Surface densities with integration ranges increased by $50 \%$. Dashes shorten as $\eta=.1, .3, .5, .7$.

where $Z(\sigma)=\sqrt{X^{2}-\sigma^{2}}$. Here again we shall compare situations where $X$ is the root of $\xi$ or is increased by $50 \%$ and even 100\%. The results are shown in Figures (77), (8) and (91). respectively. At low temperatures, a saturation of tail effects is seen in Figure (10), where Figs.(8) and (9) are fused.

A frequent observable used in astronomy is that ra-

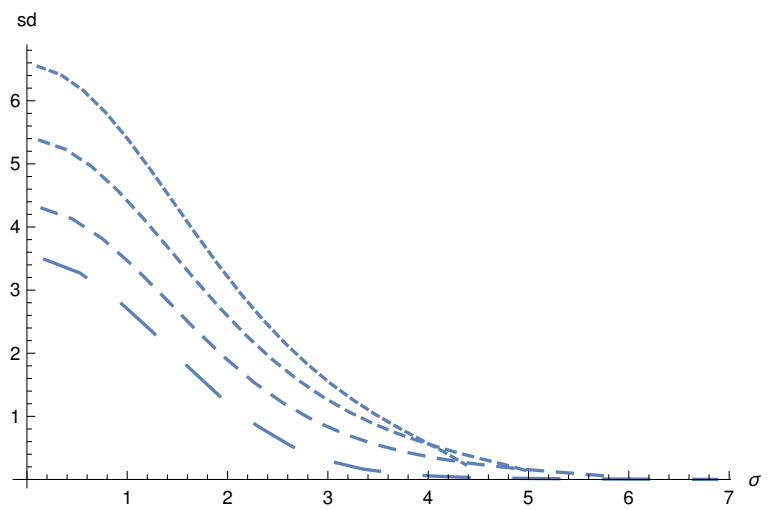

FIG. 9. Same as Fig.(8), but now with integration ranges twice the roots of $\xi$. 


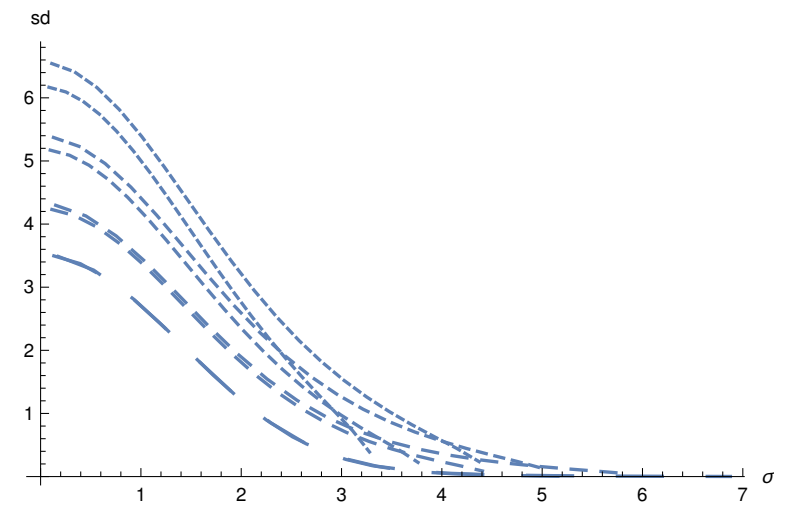

FIG. 10. Comparison of the surface densities shown in Figs. (8) and (9).

dius, $r_{c} \equiv r_{0} \tau$, where the surface density reaches half the surface density at center of the equatorial plane, namely,

$$
\int_{0}^{Z(\tau)} d \zeta \mathcal{L}_{3}\left[\xi\left(\sqrt{\tau^{2}+\zeta^{2}}\right), \eta\right]=\int_{0}^{X} d \zeta \mathcal{L}_{3}[\xi(\zeta), \eta] / 2 .
$$

Clearly, this $r_{c}$ is not observed directly. Rather, it is estimated from luminosity, decreasing from the center of the galaxy towards its edge. When the luminosity has diminished by $50 \%$, it is assumed that the same is true for the underlying dark matter surface density.

We show in Figure (11) four sets of solutions $\hat{\rho}(\tau ; \eta)$ of Eq.(32). In all four sets, the mid-density increases as a function of $\eta$. The first set, where the ranges of integrals are defined by the nodes of the $\xi$ 's, is illustrated by 8 dots, corresponding to $\eta=0, .1, .2, \ldots, .7$, respectively. It indicates that $\tau$ first increases then decreases. The second set results from integration ranges extended by $100 \%$. It contains 7 points, with $\eta=.1, .2, \ldots, .7$, and is illustrated by squares. It hints again a lack of monotonicity for $\tau$, but much less pronounced. The third set, illustrated by 7 diamonds, corresponds again to $\eta=.1, .2, \ldots, .7$. It is obtained with ranges 5 times those of the first set and exhibits a monotonic trend for $\tau$. For the fourth set, shown by triangles, with the same 7 values of $\eta$, the range extension factor is 10 . The set does not differ much from the previous one and a saturation of tail effects can be expected. Notice how the four patterns converge when $\eta$ diminishes. Actually, the lowest point of the first pattern $(\eta=0$, no extension) may be considered as belonging to all patterns as well. We list here the 7 values of $\tau$ obtained, when $\eta=.1, .2, \ldots, .7$, and the factor of integration range extension is $10:\{1.62,1.73,1.85,1.95,2.02,2.07,2.11\}$.

Now that we have at our disposal both quantities, $\sigma^{2}=$ $\left\langle p^{2} /\left(3 m^{2} N\right)\right\rangle$ and $\tau$, we can return to realistic, unscaled quantities and consider the ratio,

$$
\mathcal{R} \equiv \frac{\sigma^{2}}{G \rho_{0} r_{c}^{2}}=\frac{\sigma^{2}}{G \rho_{0} r_{0}^{2} \tau^{2}}=\frac{8 \pi \int \mathcal{L}_{5}}{5 \mathcal{L}_{3}(-1, \eta) \tau^{2} \int \mathcal{L}_{3}},
$$

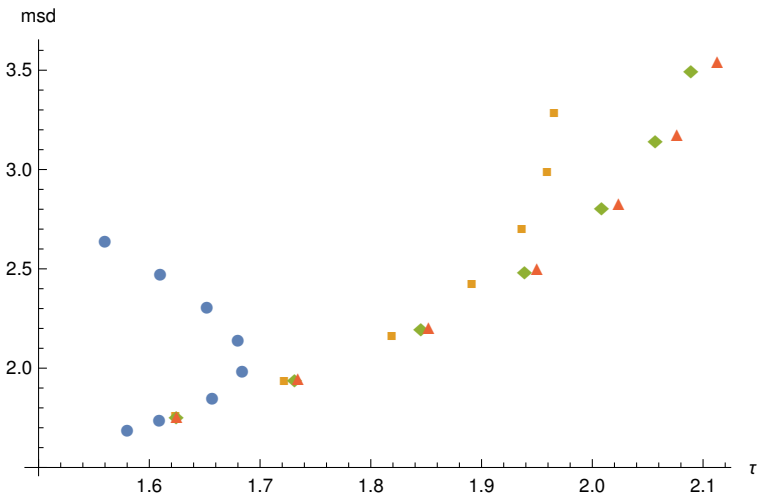

FIG. 11. Evolutions of the halved surface density and its position.

which has the advantage of being independent from $v_{0}$ and $m$. For $\eta=0$, with integral ranges confined inside $R=3.6537 r_{0}$ when we calculate surface densities, we find, numerically, $\mathcal{R} \simeq 1.60$. For $\eta=.1, .2, \ldots, .7$ we find the following, respective values, $1.59,1.55,1.53,1.52$, $1.51,1.51,1.51$. These are obtained with integral ranges extended by a factor 10, see the sequence of triangles in Fig.(11), because we want to take full tail effects into account. It is obvious that all results are compatible with a value $\mathcal{R} \simeq 1.55 \pm .05$. This privileges the King radius 14] found in the literature. Somewhat disappointing is the fact that our results for $\mathcal{R}$ show little dependence on $\eta$ and, therefore, cannot suggest a measure of the temperature of the dark matter cloud. But independence from parameters, on the other hand, may lead to solid experimental testing.

Assume that the integration range $X$ is large enough to saturate tail effects. Then, according to Eq.(29), $v_{0}^{2}=5 \sigma^{2} \mathcal{F}(\eta)$, where, $\mathcal{F}$, in a schematic notation, reads $\mathcal{F}(\eta)=\int \mathcal{L}_{3} / \int \mathcal{L}_{5}$. It is easy to tabulate. As well, according to Eq.(32), $\tau$ is a function $\mathcal{G}(\eta)$, for which we listed seven values just a few lines above. Now, according to Eq.(17), we can invert logics and obtain a formula for the mass,

$$
m^{4}=\frac{3 h^{3}}{64 \pi^{2} v_{0} G r_{0}^{2}}=\frac{3 h^{3}[\mathcal{G}(\eta)]^{2}}{64 \sqrt{5} \pi^{2} \sigma \sqrt{\mathcal{F}(\eta)} G r_{c}^{2}} .
$$

With the amplification of the integration range taken as 10 , the list of values for $\mathcal{F}(\eta)$, when $\eta=.1, .2, \ldots, .7$, reads, $\{1.19,1.02, .85, .71, .61, .53, .47\}$.

With explicit numerical constants, Eq. (34) also reads, in $\mathrm{eV}$ units for $m c^{2}$, then $\mathrm{km} / \mathrm{s}$ for $\sigma$ and $p c$ (parsec) for $r_{c}$

$$
\left(\frac{m c^{2}}{100 \mathrm{eV}}\right)^{4}=\frac{96 \cdot\{2.4,3.0,3.7,4.5,5.2,5.9,6.5\}}{\sigma /\left(10 k m s^{-1}\right)\left[r_{c} /(100 p c)\right]^{2}},
$$

where we use the typical observational scales. The string in the numerator shows the $\eta$ dependent coefficient, $[\mathcal{G}(\eta)]^{2} / \sqrt{\mathcal{F}(\eta)}$, with our usual set $\eta=.1, .2, \ldots, .7$. 
When reduced to its $1 / 4$-th power, this coefficient does not seem to be very influential.

\section{REALISTIC ILLUSTRATIONS}

Standard values in SI units for physical constants read, $k=1.3810^{-23}$, hence $k=8.610^{-5} \mathrm{eV} \mathrm{K} K^{-1}$, $h=6.610^{-34}, \quad G=6.710^{-11}, \quad c=3.010^{8}$. For the mass of dark matter particles, we shall first consider that a tolerable guess is, $m \simeq 3.610^{-34}$, hence, $m c^{2} \simeq 200 \mathrm{eV}$. If $v_{0} \simeq 10^{-4} \mathrm{c}$, a non relativistic situation, then $r_{0} \simeq 6.410^{18}$, i.e. of order $210 p c$. Recall that, $X \simeq 3.5$ for $T=0$, and that, for positive temperatures, tails may more than double the qualitative estimates of the radius. Then the cloud size may reach the $k p c$ range. Incidentally, with such estimates for $m$ and $v_{0}$, the coefficient, $\gamma \equiv .5 m v_{0}^{2} / k$, which converts $\eta$ into a temperature $T$, is of order $\simeq 0.01$. We are here, therefore, in a situation of very low temperatures if $\eta$ is kept $\leq 1$.

With different parametric assumptions, such as a lighter mass, $m \simeq 2.010^{-34} \simeq 110 \mathrm{eV} / \mathrm{c}^{2}$, and a higher reference velocity, $v_{0} \simeq 210^{-3} c$, we obtain $r_{0} \simeq 4.610^{18}$, about $150 \mathrm{pc}$, and the temperature coefficient becomes, $\gamma \simeq 2.6$. This value of $\gamma$ brings that range of $\eta$ values, studied in this paper, able to induce temperatures much closer to the CMB temperature order of magnitude.

From astronomic observations there might be some hope for measuring the center density, $\rho_{0} \equiv \rho(0)$. Typically, $\rho_{0} \sim 10^{-21} \mathrm{~kg} / \mathrm{m}^{3}$. From Eq.(4) and again a guess of $m \simeq 110 \mathrm{eV} / \mathrm{c}^{2}$, such a value of $\rho_{0}$ would return,

$$
p_{0} \equiv p_{F}(0) \sim 5.610^{-23}\left(\rho_{0}\right)^{\frac{1}{3}} \sim 5.610^{-30} .
$$

This gives $v_{0} \simeq 2.810^{4} \mathrm{~m} / \mathrm{s} \simeq 9.10^{-5} \mathrm{c}$, lower than, but not too far from the guesses made a few lines above.

More realistically, let us directly use Eq.(35) and introduce orders of magnitude $\sigma=10^{4} \mathrm{~m} / \mathrm{s}$ and $r_{c}=400 p c$, as suggested by astronomic observations. Then we obtain the string of estimates, $m c^{2}=$ $\{195,206,217,228,237,244,250\} \mathrm{eV}$, according to $\eta$.

From [4], we extract the following table of astronomic data and corresponding results,

For each astronomic object, we calculated the resulting elementary mass, seen in the right-hand-side column of the table, from Eq.(35) with the " $\eta$ coefficient" taken as 4.5 , an average value. Let $\Delta r_{c}$ and $\Delta \sigma$ be the absolute errors for $r_{c}$ and $\sigma$, respectively. Then, according to Eq. (35), we estimated the relative error for each estimated $m$ as, $\Delta m / m=\left[\sqrt{4\left(\Delta r_{c} / r_{c}\right)^{2}+(\Delta \sigma / \sigma)^{2}}\right] / 4$. As seen in the table, this elementary mass spreads between 200 and $370 \mathrm{eV}$, with error bars also spreading, between \pm 30 and $\pm 50 \mathrm{eV}$. The pattern of values and error bars is illustrated by Figure (12), their representation in increasing order being purely a convention.

\begin{tabular}{|l|c|c|c|}
\hline Object & $r_{c}(p c)$ & $\sigma(\mathrm{km} / \mathrm{s})$ & $m c^{2}(\mathrm{eV})$ \\
\hline Sextans & $630 \pm 170$ & $6.6 \pm 2.3$ & $202 \pm 32$ \\
Fornax & $400 \pm 103$ & $10.5 \pm 2.7$ & $225 \pm 32$ \\
LeoI & $330 \pm 106$ & $8.8 \pm 2.4$ & $259 \pm 45$ \\
UrsaMinor & $300 \pm 74$ & $9.3 \pm 2.8$ & $268 \pm 39$ \\
Carina & $290 \pm 72$ & $6.8 \pm 1.6$ & $295 \pm 40$ \\
Draco & $221 \pm 16$ & $9.5 \pm 1.6$ & $311 \pm 17$ \\
Bootes & $246 \pm 28$ & $6.5 \pm 1.7$ & $324 \pm 28$ \\
Sculptor & $160 \pm 40$ & $10.1 \pm 0.3$ & $360 \pm 45$ \\
Leo II & $185 \pm 48$ & $6.8 \pm 0.7$ & $369 \pm 49$ \\
\hline
\end{tabular}

TABLE I. Data from [4] and resulting elementary masses.

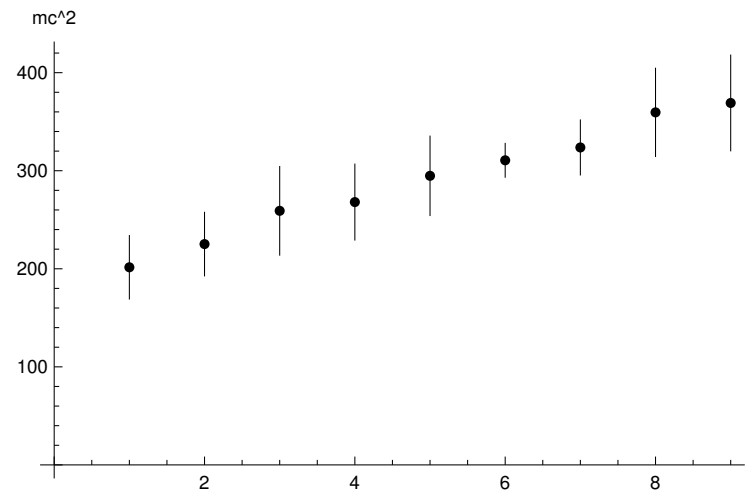

FIG. 12. Masses and error bars deduced from the astronomic data in [4]. Each integer abscissa means rank in Table I.

To compensate for this lack of precision, we implemented a $\chi^{2}$ fit for the obtained $m_{i}, m_{i}-\Delta m_{i}$ and $m_{i}+\Delta m_{i}, i=1, \ldots, 9$. This gives, $m c^{2}=290 \pm 30 \mathrm{eV}$, with $\chi^{2}=2.5$ for the central value.

A similar set of data, taken from [5], reads, with the resulting estimates for the elementary mass again from Eq.(35) and again the " $\eta$ coefficient" taken as 4.5,

\begin{tabular}{|l|c|c|c|}
\hline Object & $r_{c}(p c)$ & $v(\mathrm{~km} / \mathrm{s})$ & $\mathrm{mc^{2 }}(\mathrm{eV})$ \\
\hline CanVenat I & $564 \pm 36$ & $7.6 \pm 2.2$ & $206 \pm 16$ \\
UrsMaj I & $318 \pm 45$ & $7.6 \pm 2.4$ & $274 \pm 29$ \\
Hercules & $330 \pm 63$ & $5.1 \pm 2.4$ & $297 \pm 45$ \\
LeoT & $178 \pm 39$ & $7.5 \pm 2.7$ & $367 \pm 52$ \\
UrsMaj II & $140 \pm 25$ & $6.7 \pm 2.6$ & $426 \pm 56$ \\
LeoIV & $116 \pm 30$ & $3.3 \pm 2.8$ & $558 \pm 139$ \\
ComaBeren & $77 \pm 10$ & $4.6 \pm 2.3$ & $630 \pm 89$ \\
CanesVenat II & $74 \pm 12$ & $4.6 \pm 2.4$ & $644 \pm 99$ \\
\hline
\end{tabular}

TABLE II. Data from [5] and resulting masses.

The estimates and error bars are shown in Figure (13). After the $\chi^{2}$ processing of such 8 results, we obtain the following estimate of the mass, $m c^{2}=263 \pm 29 \mathrm{eV}$. It occurs with a disappointing $\chi^{2}=8.9$, however.

We stress, finally, that a compromise value, $m c^{2} \simeq$ 


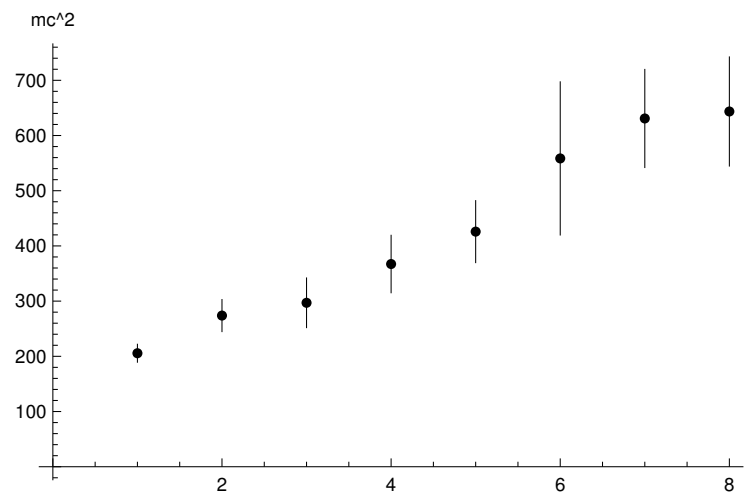

FIG. 13. Masses and error bars, astronomic data from [5]. Abscissa is rank in Table II.

$275 \mathrm{eV}$, reasonably sits in the overlap of the final error bars, $\pm 30 \mathrm{eV}$, observed from the two distinct sets of data.

\section{DISCUSSION}

Despite rather large uncertainties in astronomic data on DSG's, we have reached, however, a reasonable estimate of the "elementary dark mass", $m c^{2}=275 \pm 30 \mathrm{eV}$. This value, while a bit larger, is in a reasonable agreement with those obtained with different methods in Refs. [1-3], taking into account the experimental error bars.

Concerning the role of temperature in the range we consider, it may modify the resulting DSG-related masses by a maximum of an extra $15 \%$ amount only.

A few words of caution are in order, despite this agreement. While our theory is consistent, we found that the dispersion of obtained mass estimates between various clouds remains somewhat large, with resulting large values of $\chi^{2}$ 's. Our agreement, therefore, tends to allow for the possibility that some DSG's could be made of quasi degenerate fermions, but it should not be taken as an absolute proof of such a description for all DSG's. Another option is to turn to a different interpretation, in terms of the determination of a lower fermionic mass bound.

Indeed, assuming a thermal equilibrium for an initial stage of the formation of a dark matter cloud, then Dwarf Spheroidal Galaxies provide a rather model-independent, mass lower bound for a possible elementary dark matter fermion. Assume an initial thermal equilibrium state, like in Refs. [7-10] that we referred to in the introduction, but with a temperature low enough to allow neglecting relativistic corrections. Then let us list some distinct features that we obtain. As discussed by the authors of Ref. [8], the determination of a lower bound, from the sequence of mass determinations for the DSG's of tables I and II, is complicated by various dynamical indeterminacies. Following the choice made by [8], and for the sake of a comparison, we shall consider the central value obtained from the DSG LeoIV as a typical lower bound. This leads us to a mean value, $m c^{2} \gtrsim 550 \mathrm{eV}$ (see Table II). This is compatible with, while somewhat larger than, the result of the relativistic case $\left(m c^{2} \gtrsim 480 \mathrm{eV}\right)$ quoted in [8]. Note that we also find a consistency with the simulation-based determination, $m c^{2}$ gtrsim500 $\mathrm{eV}$, of Ref. 10]. Interestimgly, the agreement is even valid for each of our individual mass determinations for DSG's in Tables I and II. All these lie within 1 standard deviation from those quoted in [10].

The results quoted in Refs. [7, 9] are somewhat different and larger, but a comparison is then not easy, because the DSG's which are considered by [7, 9] are not the same as those that we and Refs. 8, 10] have used.

\section{CONCLUSION}

The present theory allows the calculation of basically all properties of a dark cloud, in terms of three parameters, namely an elementary particle mass $m$, a velocity scale $v_{0}$ and a temperature scale $\eta$. It suffers from a weak divergence due to density tail effects, but we have seen that many properties are not sensitive to the divergence. In fact, astronomy related observables such as the core radius $r_{c}$, and the radial velocity dispersion $\sigma$, (assumed here to be given by the luminous content of the DSG's) are shown to be free of divergence.

Note that the theoretical estimates might also suffer from an insufficient treatment of exchange terms in this mean field theory, but exchange potentials are unlikely to reach astronomic ranges.

We have seen, towards the end of Section II, that the theory provides values of the ratio, $\mathcal{R}=\sigma^{2} /\left(G \rho_{0} r_{c}^{2}\right)$, that are almost parameter independent, in particular from the temperature, and in agreement with the King radius. This may lead to some trust in the theoretical treatment.

Obviously, the particle mass $m$ is not really a parameter. Rather, it should emerge as the same physical result for all observations. Our theory easily allows, see Eq. (35), to estimate $m$ from the two traditional observables, $\sigma$ and $r_{c}$. It will be noted that the "slightly debatable" functions, $\mathcal{F}(\eta)$ and $\mathcal{G}(\eta)$, contained in Eq.(34), have values not far from $\mathcal{O}(1)$, and vary smoothly only. With the former and the latter functions taken to powers $1 / 8$ and $1 / 2$, respectively, their influence remains small.

All being considered, our results can be expressed in terms of two different fermionic mass scales, depending on the physical interpretation of the stage at which the fermionic cloud is considered to be in a non-relativistic thermal equilibrium. If one assumes that thermal equilibrium describes the present observable stage of the DSG's, then one obtains a fermion mass estimate of $m c^{2}=275 \pm 30 \mathrm{eV}$. If one rather assumes that the equilibrium corresponds to an initial stage only, followed by a 
collision-less and dissipation-less evolution, one is led to an estimated lower bound, $550 \mathrm{eV} \lesssim m c^{2}$, according to the reasoning of Refs. [8, 10]. The compatibility, or possible tension, of such determinations with other sources of astronomic observables is beyond the focus of the present work. But it obviously deserves to be studied.

As a final remark on our theory, we have not been able to design a precise way to deduce a dark cloud temperature from astronomic data, because our main result, Eq.(35), does not seem to make the elementary mass to depend on the " $\eta$ parameter" strongly enough. This problem will remain under our consideration.

\section{ACKNOWLEDGEMENTS}

We thank Patrick Valageas for remarks and suggestions on the manuscript.

* bertrand.giraud@cea.fr

† robi.peschanski@cea.fr

[1] V. Domcke and A. Urbano, JCAP 1501, no. $01, \quad 002 \quad(2015) \quad$ doi:10.1088/1475-7516/2015/01/002 arXiv:1409.3167 [hep-ph]].

[2] S. Alexander and S. Cormack, "Gravitationally bound BCS state as dark matter," JCAP 1704, no. 04, 005 (2017) doi:10.1088/1475-7516/2017/04/005 arXiv:1607.08621 [astro-ph.CO]].

[3] L. Randall, J. Scholtz and J. Unwin, "Cores in Dwarf Galaxies from Fermi Repulsion," Mon. Not. Roy. Astron. Soc. 467, no. 2, 1515 (2017) arXiv:1611.04590 [astroph.GA]].
[4] J. D. Simon and M. Geha, "The Kinematics of the Ultra-Faint Milky Way Satellites: Solving the Missing Satellite Problem," Astrophys. J. 670, 313 (2007) arXiv:0706.0516 [astro-ph]].

[5] G. Gilmore, M. I. Wilkinson, R. F. G. Wyse, J. T. Kleyna, A. Koch, N. W. Evans and E. K. Grebel, "The Observed properties of Dark Matter on small spatial scales," Astrophys. J. 663, 948 (2007) astro-ph/0703308 [ASTRO-PH]].

[6] S. Tremaine and J. E. Gunn, "Dynamical Role of Light Neutral Leptons in Cosmology," Phys. Rev. Lett. 42, 407 (1979).

[7] J. J. Dalcanton and C. J. Hogan, "Halo cores and phase space densities: Observational constraints on dark matter physics and structure formation," Astrophys. J. 561, 35 (2001) doi:10.1086/323207 astro-ph/0004381.

[8] A. Boyarsky, O. Ruchayskiy and D. Iakubovskyi, "A Lower bound on the mass of Dark Matter particles," JCAP 0903, 005 (2009) arXiv:0808.3902 [hep-ph]].

[9] C. Destri, H. J. de Vega and N. G. Sanchez, "Fermionic warm dark matter produces galaxy cores in the observed scales because of quantum mechanics," New Astron. 22, 39 (2013) doi:10.1016/j.newast.2012.12.003 arXiv:1204.3090 [astro-ph.CO]].

[10] S. Shao, L. Gao, T. Theuns and C. S. Frenk, "The phase space density of fermionic dark matter haloes," Mon. Not. Roy. Astron. Soc. 430, 2346 (2013) arXiv:1209.5563 [astro-ph.CO]].

[11] D. R. Hartree, Proc. R. Soc. London A113, 621 (1928).

[12] V. A. Fock, Z. Phys. 61, 126 (1930).

[13] L. H. Thomas, Proc. Cambridge Philos. Soc. 23, 542 (1927). E. Fermi, Z. Phys. , 48, 73 (1928). J. C. Slater, Phys. Rev , 81, 385 (1951).

[14] J. Binney and S. Tremaine "Galactic Dynamics" Princeton University Press ed. (1987, Second Edition, 2008) 